\title{
Neural mechanisms underlying the income-achievement gap: The role of the ventral visual stream
}

\author{
Maya L. Rosen ${ }^{\text {a, }, \text { Lucy A. Lurie }}{ }^{\mathrm{b}}$, Kelly A. Sambrook ${ }^{\mathrm{a}}$, Andrew N. Meltzoff ${ }^{\mathrm{c}, \mathrm{d}}$, \\ Katie A. McLaughlin ${ }^{a}$ \\ ${ }^{\text {a }}$ Harvard University, Department of Psychology, Cambridge, MA, USA \\ ${ }^{\mathrm{b}}$ University of North Carolina, Chapel Hill, Department of Psychology \& Neuroscience, Chapel Hill, NC, USA \\ ${ }^{\mathrm{c}}$ University of Washington, Institute for Learning \& Brain Sciences, Seattle, WA, USA \\ ${ }^{\mathrm{d}}$ University of Washington, Department of Psychology, Seattle, WA, USA
}

\section{A R T I C L E I N F O}

\section{Keywords:}

Socioeconomic status (SES)

Executive function

Academic achievement

Visual association cortex

Cognitive stimulation

fMRI

\begin{abstract}
A B S T R A C T
Children from low-socioeconomic status (SES) households on average exhibit lower academic achievement than their higher-SES peers. We investigated a novel hypothesis that differences in early-developing sensory networks - specifically the ventral visual stream (VVS), which is involved in processing visual stimuli-contribute to SES-related disparities in executive functions (EF) and academic outcomes. We used fMRI to investigate SESrelated differences in neural function in children (6-8 years, $n=62$ ) during two attentional tasks involving attention to visual information: cued attention and memory-guided attention. Recruitment of VVS during both tasks was associated with EF and academic achievement, and SES-related differences in VVS activation during cued attention were marginally explained by differences in cognitive stimulation. VVS activation during cued attention mediated SES-related differences in academic achievement. Finally, the link between VVS activation during both tasks and academic achievement was mediated by differences in EF. We extend previous work by highlighting that: (i) early-developing visual processing regions play a role in supporting complex attentional processes, (ii) childhood SES is associated with VVS function, which is explained in part by SES-related differences in cognitive stimulation and (iii) provide preliminary evidence that individual differences in VVS function may play a role in the emergence of the income-achievement gap.
\end{abstract}

\section{Introduction}

Children from lower socioeconomic status (SES) households exhibit lower academic achievement, on average, than their higher-SES peers. This pattern is known as the income-achievement gap. Recent studies have shown that this gap has been stable (Hanushek et al., 2019) or even grown over the last 50 years (Reardon, 2013). However, it remains unclear why this gap exists. Here, we seek to investigate environmental, neural, and cognitive mechanisms explaining this gap. We propose that SES is associated with cognitive stimulation in the early home environment, which influences development of visual processing regions including the ventral visual stream (VVS). We further propose that the development of VVS, in turn, scaffolds development of higher-order cognitive skills including executive functions (EF) and that this plays a critical role in explaining the income-achievement gap (Fig. 1A). We test this model in a longitudinal study of children aged 6-8 years.

\subsection{Socioeconomic status and ventral visual stream}

Growing evidence indicates that childhood SES is associated with differences in both brain structure and function (Finn et al., 2017; Mackey et al., 2015; McDermott et al., 2019; Noble et al., 2015; Rosen et al., 2018). Given that children raised in low-SES households also tend to exhibit lower performance on tests of higher-order cognitive functions, including attention, memory, EF, and language (Lawson et al., 2018; Lipina et al., 2013; Noble et al., 2005, 2007; Rowe, 2008), current research has focused largely on the neural networks that support these complex cognitive functions (Noble et al., 2007; Noble et al., 2005; Romeo et al., 2018; Rosen et al., 2012). However, many studies have also found SES-related differences in the structure and function of VVS-a set of brain regions involved in processing visual stimuli (Finn et al., 2017; Leonard et al., 2019; Mackey et al., 2015; Noble et al., 2015; Rosen et al., 2018). The majority of these findings emerged from

\footnotetext{
* Corresponding author.

E-mail address: mayalrosen@fas.harvard.edu (M.L. Rosen).
} 
exploratory analyses that did not specifically hypothesize these SES-related differences in VVS. Rather, VVS differences often emerged among many other brain areas that show SES-related differences in structure or function. As such, there has been little attempt to interpret why SES might be linked to early-developing sensory processing regions or how neural differences in VVS might contribute to SES-related disparities in higher-order cognitive development.

We have proposed that development of VVS may be influenced by early environmental experiences which tend to occur in higher rates among children from low-SES families, including lower levels of cognitive and social stimulation (Rosen et al., 2019). A recent conceptual model posited that VVS scaffolds the development of higher-order cognitive abilities through feedforward and feedback connections with the prefrontal cortex (PFC) by sending information that is complex and overlapping (Amso and Scerif, 2015). Here, we build on this framework, proposing that cognitive stimulation could influence development of VVS and in turn, the PFC and higher order cognitive abilities. Specifically, cognitive stimulation in the context of caregiver interactions may support children's ability to regulate attention to relevant visual cues and to identify and categorize visual information. For example, a caregiver can highlight the features of an object that help children learn to categorize stimuli, such as directing attention to critical visual features that distinguish between basic-level categories (e.g., a cat's pointed ears, size, or eye shape vs. those of a dog). These processes require interactions between VVS and PFC through the regulation of attention to stimulus features cued by a caregiver to facilitate formation of representations of semantic categories. Differences in cognitive stimulation may impact the development of VVS, particularly during early periods of rapid growth in children's semantic knowledge, associative memory, and selective attention. We have further proposed that functional differences in visual processing regions observed in children from low-SES backgrounds may contribute to SES-related differences in EF (Lawson et al., 2018; Rosen et al., 2019), which in turn may explain SES-related disparities in academic achievement (Finn et al., 2017; Rosen et al., 2020). In the present preliminary study, we examine cognitive stimulation as a potential malleable environmental mechanism explaining the association between SES and VVS function.

\subsection{Cued attention, memory-guided attention and academic achievement}

Children readily use both visually present cues and their memories to effectively direct attention (Brooks et al., 2019; Dixon et al., 2010; Nussenbaum et al., 2019; Perchet and García-Larrea, 2000; Senju et al., 2004). We focus on functional activation in VVS during two tasks that require attention to visual information: (i) cued attention-the ability to use an external visual cue to direct attention to a specific location in the environment, and (ii) memory-guided attention-which requires using memories from past experience to direct attention to the current environment. Both of these processes are associated with academic performance in children (Rosen et al., 2019; Rothbart et al., 2007) and typical academic activities engage a broad set of complex processes including acquisition, memory, and directed attention for perceptual information. Here, we investigate how individual differences in the functional recruitment of VVS during cued and memory-guided attention may be a mechanism explaining the income-achievement gap. Because work in adults suggests greater activation in VVS during cued compared to memory-guided attention (Rosen et al., 2016), we expect that there may be stronger associations of VVS activation during cued attention with SES, cognitive stimulation, EF and academic achievement than during memory-guided attention.

\subsection{Executive function and academic achievement}

EF are a set of cognitive skills important for goal-directed adaptive functioning (Blair and Razza, 2007). Children growing up in low-SES environments tend to exhibit lower performance on attentional and $\mathrm{EF}$ tasks (Lawson et al., 2018; Mezzacappa, 2004; Rosen et al., 2020; Rosen et al., 2019), cognitive processes that are strongly related to academic success in Western schools (Blair and Razza, 2007; Rabiner et al., 2004, 2016; Samuels et al., 2016). Indeed, differences in EF have been shown to play an important role in explaining the income-achievement gap (Finn et al., 2017; Rosen et al., 2020). Our model and others posit that development of VVS scaffolds development of the PFC, which in turn may influence development of EF (Amso and Scerif, 2015; Rosen et al., 2019; Fig. 1A). We therefore test the hypothesis that differences in EF may be a mechanism linking VVS function with academic achievement.

\subsection{Present study}

The present study had four overarching goals focused to understand the environmental, neural, and cognitive mediators of the achievement gap. The first was to examine whether SES was related to VVS recruitment during cued and memory-guided attention, and whether cognitive stimulation mediated this association. The second was to investigate whether VVS recruitment was associated with differences in EF performance. The third aim was to examine whether VVS function was associated with academic achievement and to determine whether differences in EF accounted for this association. Our final goal was to test the hypothesis that SES-related differences in functional activation of VVS contribute to the income-achievement gap.

This study focuses on an important time window in development-young children beginning school, which is a crucial period at which to understand the relation between SES and academic achievement. We collected data at two time points to capture both cognitive stimulation in the home and academic achievement when children are in the early, foundational grades in school. At the first time point, we collected demographic data including SES along with an in-depth home visit designed to assess cognitive stimulation. Approximately 18 months later, children came to the laboratory to complete neuroimaging as well as tests of EF and academic achievement. Finally, low-SES is associated
A

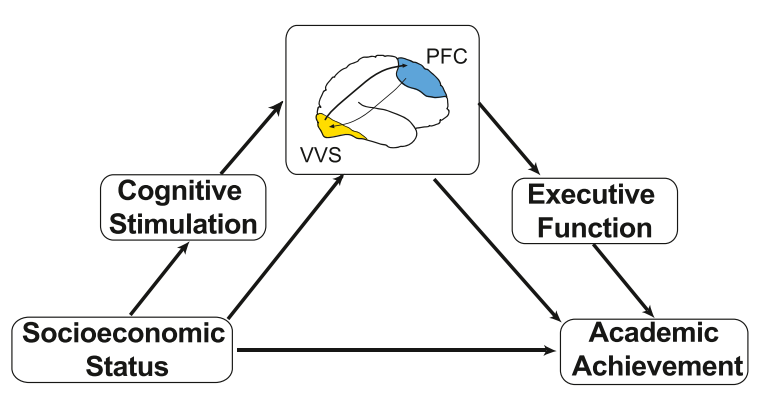

B

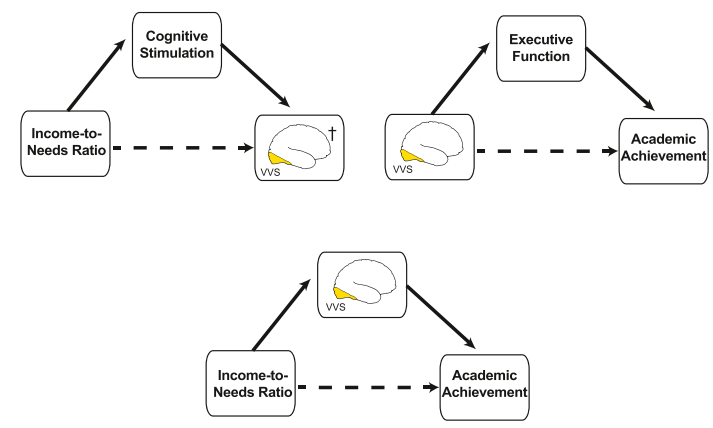

Fig. 1. (A) Conceptual model, (B) illustration of significantly mediations. ${ }^{\dagger}$ indicates marginally significant mediations. 
with increased risk for other forms of adversity, such as experiencing violence (McLaughlin et al., 2012). To isolate SES-related differences in neural function and academic achievement that are not driven by these experiences, we control for violence exposure in all analyses including SES or cognitive stimulation as predictors.

\section{Methods}

\subsection{Participants}

Seventy-two participants aged 6-8 years $(M=7.02 ; S D=0.46 ; 36$ female) who had data at both timepoints participated. The sample was originally recruited in Seattle, WA between January 2016 and September 2017 as part of a larger study on the associations between the home environment and cognitive development $(n=99$, Rosen et al., 2020, Lurie et al., 2021). The Institutional Review Board of the University of Washington approved all procedures. Participants were compensated and written informed consent was obtained from legal guardians. Youths provided verbal assent. The race and ethnicity of the families closely matched the demographics of the greater Seattle area (65.2\% White, $12.5 \%$ Black, 4.2\% American Indian or Alaska Native, $11.1 \%$ Asian, $2.7 \%$ Native Hawaiian or Pacific Islander, $4.2 \%$ Other; with $11.1 \%$ identifying as Hispanic or Latino).

\subsection{Procedure}

Families completed a comprehensive assessment of family SES and the home environment approximately 18 months prior to the assessments of academic achievement and the fMRI sessions (T1, $M=17.45$ months before $\mathrm{T} 2, S D=4.03)$. A total of 72 children $(72.72 \%)$ from the original sample participated in both the assessment of academic achievement and the neuroimaging tasks. Nine participants were excluded due to having more than $20 \%$ high-motion volumes during the fMRI scan, and one participant was excluded due to lack of behavioral data from the scan session. The final analytic sample included 62 children $(M=7.04 ; S D=0.48$; 31 female). Motion was negatively associated with age but was not significantly associated with any of our variables of interest (see Table 2).

Participants made two laboratory visits. In the first (T2), participants completed encoding for the cued and memory-guided attention tasks, EF tasks, tests of academic achievement, and a mock scan. The second visit (within 1 week) involved the fMRI scan (T3). Due to travel and scheduling constraints, one male participant completed both $\mathrm{T} 2$ and T3 on the same day (separated by about $3 \mathrm{~h}$ ) and one female participant completed the T2 and T3 one month apart. None of the statistical results meaningfully change if we exclude the subjects with abnormal intervals between encoding and test, and we retain them in all analyses.

\subsection{Measures}

\subsubsection{Socioeconomic status}

SES was measured using the income-to-needs ratio (INR) which captures the amount of annual income that a family earns relative to the federal poverty line for a family of that size at T1.

Median INR was 4.75 with $8 \%$ of participants living in poverty (income to needs less than 1 ) and $17.7 \%$ of participants living at less than twice the poverty line (Fig. 2). Income at T1 and T2 were highly correlated ( $r=0.914, p<.001$; see Supplemental material). INR values were log-transformed for all analyses, which is a common practice in developmental studies because SES associations with cognitive and neural development are non-linear and strongest at the lower end of the SES distribution (Noble et al., 2015; Rosen et al., 2018). In all analyses, the association between SES and neural and behavioral outcomes was stronger when using a log-transformed INR compared to linear INR.

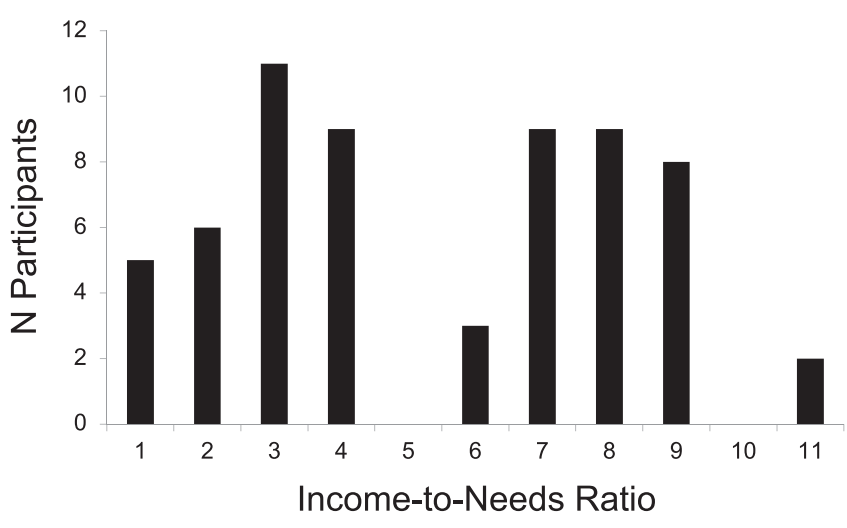

Fig. 2. Histogram of the income-to-needs ratio for the final sample.

\subsubsection{Cognitive stimulation}

Two experimenters visited the family home at $\mathrm{T} 1$ to assess cognitive stimulation of the home using a standard measure, the Home Observation of the Environment (HOME), Early Childhood version (Bradley et al., 2001). We hypothesized that cognitive stimulation in the context of caregiver interactions scaffolds development of VVS and higher-order cognitive function. Therefore, we created a composite score from the HOME interview using items that specifically focus on language stimulation and caregiver engagement and involvement in the child's learning. These include parental support of the child's learning, paren$\mathrm{t}$-child interactions, and linguistic stimulation from the caregiver (see Supplementary materials for specific items). This composite measure includes only elements of cognitive stimulation that reflect parent-child interactions and activities that help support learning and excludes items that simply reflect objects that families own (e.g., books, toys, etc.). This composite measure best captures the experiences we predict are important for development of VVS, EF, and academic achievement (Rosen et al., 2019). Confirmatory factor analysis indicated that our model of cognitive stimulation fit the data well $\left(X^{2}(35)=27.169\right.$, $p=.925$; RMSEA $<0.001,90 \%$ CI $[0.000,0.045], p=.963$; CFI $=1.00)$.

\subsubsection{Violence exposure}

To assess exposure to violence, parents completed the Violence Exposure Scale for Children-Revised (VEX-R; Fox and Leavitt, 1995) in a format adapted for parent at T1. This variable was used as a covariate (see Supplemental materials).

\subsubsection{Attention tasks}

The two attention tasks were adapted for children from a set of cued attention and memory-guided attention tasks developed for adults and have been used previously in young children (Rosen et al., 2019; Rosen et al., 2018; Fig. 3). A set of eight objects familiar to children (e.g., cup, boot, pillow) were used as targets. These images were split into two lists (List A and List B). The lists were counterbalanced across participants such that half of the subjects received List $A$ as targets for cued attention and List $B$ as targets for memory-guided attention and the other half the reverse. The order of these two tasks was also counterbalanced across participants. Twelve familiar objects (e.g., tree, apple) served as distractors for both tasks and never appeared as targets. The two encoding phases took place during T2, the behavioral laboratory visit. The two test phases took place during T3, the fMRI lab visit.

2.3.4.1. Cued attention encoding phase. During the encoding phase for the cued attention task, participants viewed a word and picture (e.g. cup) presented at the center of the screen along with an audio cue (e.g. "cup"). The picture remained on the screen for $1500 \mathrm{~ms}$ and the participants saw each picture 8 times for a total of 32 trials (Fig. 3A). This ensured that the viewing time per image and number of exposures per 
A Encoding for Cued Attention Task

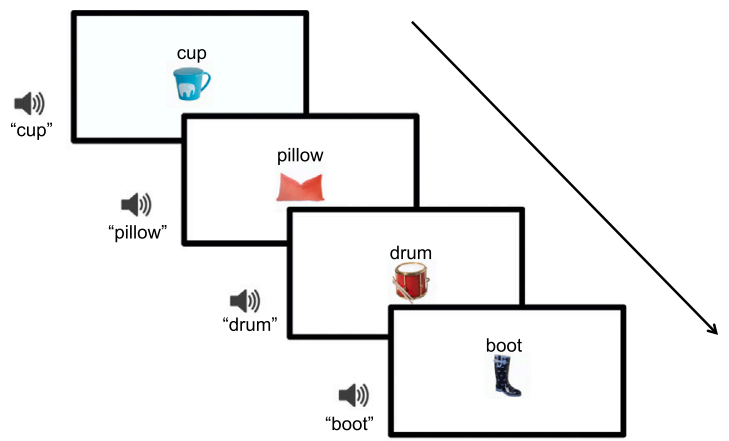

C

\section{Encoding for Memory-Guided Attention Task}

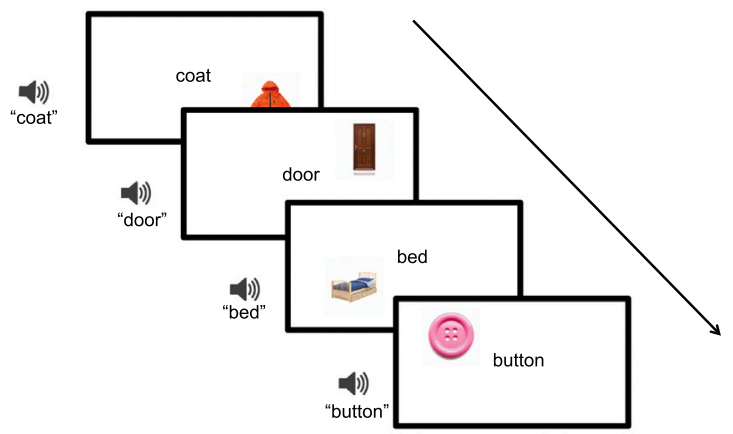

B

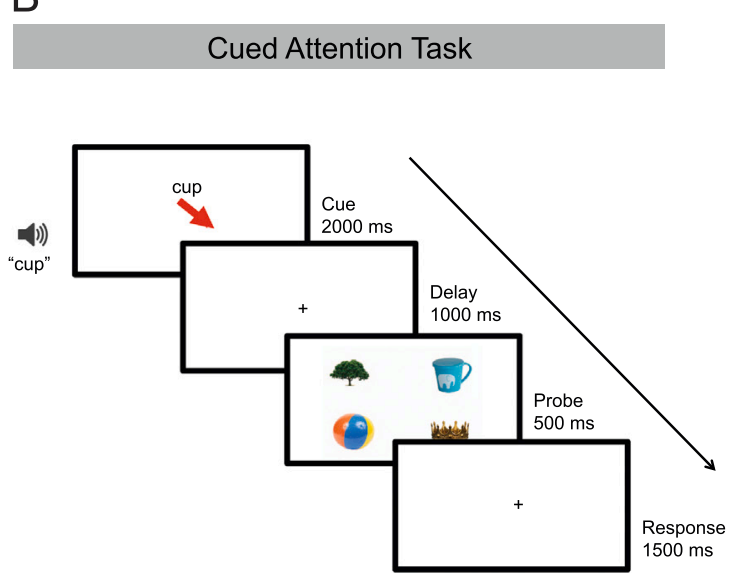

D

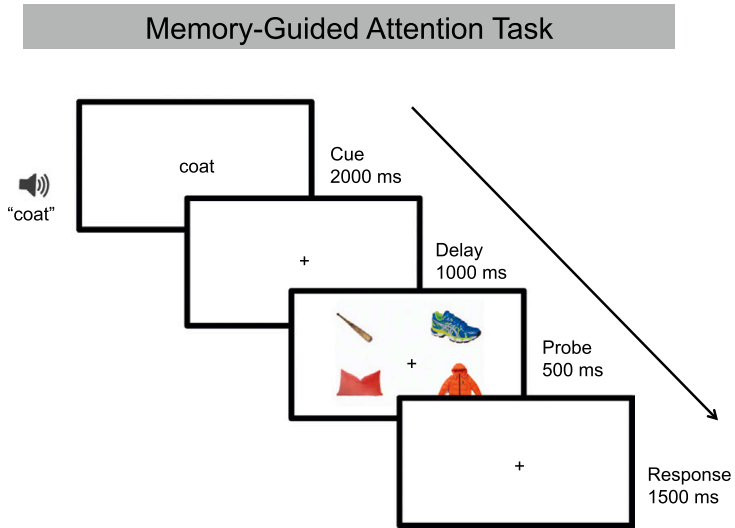

Fig. 3. Cued and memory-guided attention tasks. During the encoding phase of the cued attention task (A), participants simply viewed the objects that would appear as targets in the test phase. During the test phase for the cued attention task (B), which occurred in the scanner, a word and an arrow appeared on the screen along with an audio cue of that word. Participants were told to direct their attention to the location where the arrow was pointing. After a brief delay, four pictures appeared, one in each quadrant. Participants made a button response to indicate if the picture appeared at the cued location or at a different location. During the encoding phase of the memory-guided attention task (C), which occurred outside of the scanner, participants learned object-location pairings. During the test phase, which occurred in the scanner (D), a word appeared on the screen along with an audio cue of that word. Participants were instructed to pay attention the location where the picture belongs. After a brief delay, four pictures appeared, one in each quadrant. Participants made a button response to indicate if the picture appeared at the cued location or at a different location. Responses were taken during the probe presentation and the response period. In the example pictured here, the target appeared at the cued location. Across both tasks, the target appeared in the cued location on $50 \%$ of trials and in a different location on the other $50 \%$ of trials.

image was matched across the two tasks. During the encoding phase, participants saw each object for total of $12 \mathrm{~s}$, the same amount of time each object was seen during encoding for the memory-guided attention task.

2.3.4.2. Memory-guided attention encoding phase. The encoding phase for the memory-guided attention task consisted of two parts in which participants learned to bind an object with a spatial location (Fig. 3C). During encoding phase 1 , participants were presented with an object image (e.g. button) located in one of the four quadrants along with an audio cue (e.g. "button"). Participants were instructed to "look at the screen and try to remember where the picture goes." The word and the image were presented simultaneously for $2000 \mathrm{~ms}$. Each image was presented four times for a total of 16 trials with each picture only ever appearing in one of the four quadrants. During encoding phase 2, participants viewed and heard the word cue $(2000 \mathrm{~ms})$ without the image followed by a blank screen for $1000 \mathrm{~ms}$. Participants were instructed to try to touch the screen where the picture should go before the picture appeared. Then the picture appeared in the correct location for $1000 \mathrm{~ms}$. This additional encoding was designed to aid in the binding of the picture with the spatial location. During this phase, each object was presented four times for a total of 16 trials. Across the two encoding phases, participants saw each object for total of $12 \mathrm{~s}$, the same amount of time each object was seen during encoding for the cued attention task.

2.3.4.3. fMRI tasks. Before scanning, participants were given a brief explicit memory test to ensure they remembered the locations of the four objects for the memory-guided attention task. The experimenter presented the child with a blank computer screen and asked the child to point to the location where each object goes (e.g. "Where does the button go?"). Objects were presented in random order. All participants except 5 ( 2 female) remembered all four locations on the first try. The participants who did not remember all four locations were reminded of the location of each object by the experimenter, followed by an additional explicit memory test. After the reminder, all participants remembered all four object-location pairings.

To ensure that participants understood the task, they first completed one practice run before scanning. To create a tactile reminder of which hand to use for each button press, we placed a sticker on the hand that should be used to make a button press to indicate that the picture appeared in the cued location (valid cue). Before each run, we asked for a verbal response about which hand corresponded to which response 
("sticker hand" or "no sticker hand"). Finally, to increase motivation, participants chose several prizes that they would earn upon completion of the scan. Before each run we told them which prize they were "playing for," and which prize they had won after each run.

Participants completed two runs of the task in the scanner. Runs consisted of alternating blocks of eight trials of cued and memoryguided attention, the order of which were counterbalanced across participants. Both tasks consisted of a cue phase $(2000 \mathrm{~ms})$, a delay $(1000 \mathrm{~ms})$, a probe $(500 \mathrm{~ms})$ and a response period $(1000 \mathrm{~ms})$. Responses were taken during both the probe and response periods, giving participants a total of $1500 \mathrm{~ms}$ to respond. In any given block, for half of the trials, the target appeared in the cued location (valid cue) and for the other half, the target appeared in a different location (invalid cue) with equal probability that the target would appear in one of the other three non-cued locations. Participants made a button press with one hand if the target appeared at the cued location and with the other hand if the target appeared at a different location. Before going into the scanner, participants were asked which hand they used if the picture appeared in the "right spot" and which hand to use if the picture appeared in a "different spot." Participants were also asked again before beginning each run of the task. Each run consisted of three blocks of cued attention and three blocks of memory-guided attention as well as blocks of fixation.

In the cued attention task, participants saw a word on the screen and also heard the word. An arrow was presented at the center of the screen that pointed to the location to which the participant should attend. Participants were instructed to use the arrow to pay attention to the spot on the screen where the picture should appear. After the delay, participants saw an array of four objects and responded whether the target appeared at the cued location or at a different location with a button press (Fig. 3B).

In the memory-guided attention task, participants saw a word on the screen and also heard the word through headphones. Participants were instructed to use their memory to pay attention to the spot on the screen where the picture should appear. After the delay, participants saw an array of four objects and indicated whether the target appeared at the correct (remembered) location or in a different location with a button press (Fig. 3D). See Supplemental materials for additional details.

Performance was assessed using the sensitivity index $\left(d^{\prime}\right)$, which was calculated using the following formula:

$d^{\prime}=\mathrm{z}($ hit rate $)-\mathrm{z}($ false alarm rate $)$

where $\mathrm{z}$ is the standardized score and $d^{\prime}$ provides a metric of sensitivity to detect whether a target appears in a cued location.

\subsubsection{Executive function}

At T2, participants also completed three standard tests EF including the backwards digit span (BDS) as a test of working memory, Simon Says as a test of inhibitory control, and a dimensional change card sort (DCCS) task as a test of cognitive flexibility. These tasks are wellestablished and we have described them previously (Carlson and Meltzoff, 2008; Rosen et al., 2020; Zelazo, 2006; see Supplemental materials). We created an EF composite score by taking the mean of the standardized final score for the BDS, Simon Says, and DCCS.

\subsubsection{Academic achievement}

Three subtests of the Woodcock-Johnson IV Tests of Achievement (WJ-IV) were used as assessments to compute the Academic Skills Cluster (Schrank et al., 2015): Letter-Word Identification, Spelling, and Calculation (see Supplemental materials).

\section{4. fMRI image acquisition, preprocessing, and analysis}

See Supplemental materials for fMRI image acquisition and preprocessing.

\subsection{1. fMRI model construction}

We constructed models using correct trials only. We modeled all levels of the task (cue, delay, and probe) for each condition using a boxcar function for each block. Three blocks of fixation of $10 \mathrm{~s}$ each were intermixed with the task blocks and these fixation blocks were modeled implicitly. Individual-level estimates of BOLD activity were submitted to group-level random effects models of cued and memoryguided attention, each compared to baseline (fixation) and additionally constructed contrasts of cued attention $>$ memory-guided attention and memory-guided attention $>$ cued attention. Incorrect trials were included in the model as a regressor of non-interest.

\subsubsection{Correction for multiple comparisons}

To correct for multiple comparisons in whole-brain analyses, AFNI's 3dClustSim program was used to calculate an appropriate cluster size threshold. Recent simulations demonstrate elevated risk of false positives using standard cluster-level correction approaches (Eklund et al., 2016). Recent updates to AFNI's 3dFWHMx and 3dClustSim utilize a spatial autocorrelation function (ACF), which more accurately estimates the spatial smoothness to reduce the risk of false positives. The ACF option in 3dFWHMx was used to estimate the spatial smoothness of residuals from the individual-level analyses. The average ACF will then be used with $3 \mathrm{dClustSim}$ to calculate the appropriate cluster size threshold with 10,000 Monte Carlo simulations. We used a voxel-wise threshold of $\mathrm{p}<.01$ and family-wise error corrected cluster-level threshold of $p<.05$.

\subsection{Statistical analysis}

We used multiple regression to examine the associations between INR and cognitive performance, and INR and academic achievement. All analyses controlled for age and sex. Analyses exploring associations with INR and cognitive stimulation also controlled for violence exposure. These analyses were performed in R. All parameter estimates are reported in standardized units.

To examine the associations between INR, cognitive stimulation, and academic achievement with neural function, we took two approaches: a whole-brain analysis and a region of interest (ROI) analysis in VVS regions that process complex visual stimuli.

\subsubsection{Whole-brain analysis}

We first examined the pattern of brain activation for each task compared to baseline (see Supplemental materials for contrast of cued vs. memory-guided attention). This allows us to establish the pattern of functional activation during these tasks in a sample of young children.

Next, for analyses focused on associations with the environment and academic achievement, we focused on the contrasts between each task compared to baseline. To do this, we estimated separate models with mean-centered regressor for log income-to-needs (INR), cognitive stimulation, and academic achievement for the contrasts of interest (cued attention $>$ baseline and memory-guided attention $>$ baseline), with every model controlling for age at the time of scanning and sex. All models with INR or cognitive stimulation as a predictor or outcome also included violence exposure as a covariate.

\subsubsection{Region of interest analysis}

We created functionally defined ROIs by masking the task-related activation (both cued and memory-guided attention) versus baseline in the entire sample with a mask of the temporal-occipital fusiform gyrus and lateral occipital cortex, inferior division ( $20 \%$ threshold) from the Harvard-Oxford cortical atlas in FSL to create two separate (left and right) VVS ROIs. For the ROI-based approach, we used multiple regression to examine the associations between INR and neural activation in VVS ROIs and the associations between VVS activation and academic achievement. Results were false discovery rate (FDR) corrected at the level of hypothesis (e.g. we conducted two separate tests of each 
hypothesis focused on VVS activation-one in left and one in right VVS - and corrected for these two tests of the same hypothesis).

\subsubsection{Mediation analyses}

Finally, we used a standard test of statistical mediation that estimates the significance of indirect effects using a bootstrapping approach that provides confidence intervals for the indirect effects (Hayes, 2013). Mediation analyses were conducted in SPSS 26.01. Confidence intervals that do not include 0 are considered evidence for statistically significant indirect effects. We tested three sets of indirect effects. The first tested whether INR has an indirect effect on VVS activation through cognitive stimulation. The second tested whether VVS recruitment had an indirect effect on academic achievement through EF. The third tested whether INR had an indirect effect on academic achievement through VVS recruitment. Modern approaches to statistical mediation advocate for estimating indirect effects regardless of whether each of the a, b, and c paths are statistically significant (Hayes, 2009; MacKinnon et al., 2007; MacKinnon et al., 2000; Shrout and Bolger, 2002; Zhao et al., 2010); as such, we estimated the indirect effects for each of our hypothesized pathways, even if the individual paths were not statistically significant.

\section{Results}

Descriptive statistics are presented in Table 1. Bivariate correlations between all study variables are presented in Table 2. Summaries of full regression models are in Supplemental Table 1.

\subsection{Overall behavioral performance}

\subsubsection{Cued attention and memory-guided attention performance}

Children performed well on both tasks but demonstrated higher performance on the cued attention $(M=1.24, S D=1.08)$ compared to memory-guided attention task $(M=1.06, S D=1.12 ; t(61)=3.15$, $p=.003)$.

\subsection{Overall neural activation}

\subsubsection{Cued and memory-guided attention}

During cued attention, we found widespread activation in both the frontoparietal network-including left middle frontal gyrus (MFG), bilateral intraparietal sulcus (IPS), dorsal anterior cingulate (dACC), and superior parietal lobule, and VVS-encompassing regions in lateral occipital cortex and ventral temporal cortex, including fusiform gyrus. This contrast also revealed activation in the anterior insula, precentral gyrus, and superior temporal sulcus (Fig. 4A). The contrast of memoryguided compared to baseline revealed largely the same pattern of activation as cued attention (Fig. 4B). See Supplemental materials for contrast of Cued vs. Memory-Guided Attention.

\subsection{SES, cognitive stimulation, and VVS recruitment}

We next asked whether INR was related to VVS recruitment during

Table 1

Descriptive Statistics.

\begin{tabular}{lll}
\hline Variable & M (SD) & Range \\
\hline Age & $7.04(0.48)$ & $6.13-8.11$ \\
Sex & $0.5(0.5)$ & $0-1$ \\
Violence exposure & $2.89(3.88)$ & $0-20$ \\
Log Income-to-Needs & $1.25(1.03)$ & $-2.54-2.35$ \\
Cognitive Stimulation & $8.71(1.41)$ & $3-10$ \\
Academic Achievement & $100.61(13.06)$ & $71-141$ \\
Left VVS (Cued) & $1.01(0.83)$ & $-0.48-2.71$ \\
Right VVS (Cued) & $1.01(0.81)$ & $-0.42-2.79$ \\
Left VVS (MGA) & $0.707(0.78)$ & $-0.633-2.6$ \\
Right VVS (MGA) & $0.703(0.74)$ & $-0.82-2.76$ \\
\hline
\end{tabular}

cued and memory-guided attention, and whether this association was explained by cognitive stimulation in the home environment.

\subsubsection{INR and cognitive stimulation}

Consistent with previous studies from which the present sample is a subsample, we found that INR was positively associated with cognitive stimulation in the home $(\beta=0.433, p=.001$; Fig. $5 \mathrm{~A})$.

\subsubsection{INR and attention performance}

INR was positively associated with performance on both attention tasks. Specifically, INR was positively associated with $d^{\prime}$ on both the cued $(\beta=0.411, p<.001)$ memory-guided attention $(\beta=0.423$, $p=.002)$ tasks.

\subsubsection{INR and neural activation}

We examined whole-brain models with income-to-needs as a predictor for the contrasts of cued attention $>$ baseline and memory-guided attention $>$ baseline. We found a significant positive association between INR and activation during the cued attention task in the left inferior frontal gyrus extending into anterior insula, left putamen, and left parahippocampal gyrus (Fig. 6; Table 3). We found no associations between INR and activation in the whole brain analysis during the memory-guided attention task.

In ROI analysis of the VVS, we found a trend toward a positive association between INR and recruitment in left and right VVS $(\beta=0.235$, $p=.077 ; \beta=0.229, p=.077$; Fig. 9A) during cued attention. There was no association between INR and activation in left or right VVS during memory-guided attention ( $\beta=0.129, p=.317 ; \beta=0.152, p=.317$ ).

\subsubsection{Cognitive stimulation and neural activation}

Whole brain models with cognitive stimulation as a predictor during cued attention > baseline and memory-guided attention > baseline revealed no clusters with a significant association between cognitive stimulation and neural activation during either task.

We next tested whether cognitive stimulation was associated with activation in VVS ROIs. The association between cognitive stimulation and activation in right VVS during cued attention was significant but become trend-level after FDR-correction $(\beta=0.238, p=.104$; Fig. $5 \mathrm{~B})$ and was also marginal in left VVS $(\beta=0.202, p=.104)$. There was no significant association between cognitive stimulation and activation in left or right VVS during memory-guided attention $(\beta=0.095, p=.440$; $\beta=0.138, p=.440$ ).

\subsubsection{Cognitive stimulation as a mediator of the association of INR and} VVS activation

We next tested the hypothesis that cognitive stimulation mediates the association between income and activation in VVS (Fig. 5C). We found a marginal indirect effect of income on activation in the right VVS during cued attention through cognitive stimulation (95\% CI: [-.010,.183], 90\% CI: [.002,.160]; Table 4). No other significant indirect effects were found.

\subsection{INR, VVS recruitment, and EF}

Our next question was whether VVS recruitment during cued and memory-guided attention was associated with EF and explained the link between INR and EF.

\subsubsection{INR and $E F$}

INR was positively associated with EF such that higher income-toneeds was associated with higher scores on the EF composite $(\beta=0.286, p=.019)$.

\subsubsection{VVS function and $E F$}

We then examined whether neural activation in VVS ROIs was associated with EF. Neural activation in left and right VVS during cued 
Table 2

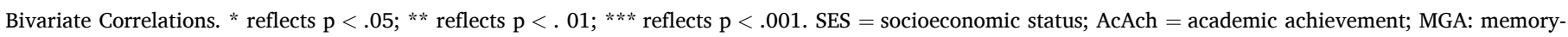

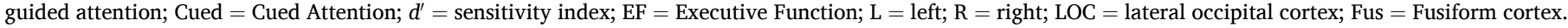

\begin{tabular}{|c|c|c|c|c|c|c|c|c|c|c|c|c|c|c|}
\hline & Age & Sex & Violence & Motion & SES & $\begin{array}{l}\text { Cog } \\
\text { Stim }\end{array}$ & Cued d' & $\begin{array}{l}\text { MGA } \\
\text { d' }\end{array}$ & $\mathrm{EF}$ & Ac Ach & $\begin{array}{l}\text { L VVS } \\
\text { Cued }\end{array}$ & $\begin{array}{l}\text { R VVS } \\
\text { Cued }\end{array}$ & $\begin{array}{l}\text { L VVS } \\
\text { MGA }\end{array}$ & $\begin{array}{l}\text { R VVS } \\
\text { MGA }\end{array}$ \\
\hline \multicolumn{15}{|l|}{ Age } \\
\hline Sex & 0.043 & & & & & & & & & & & & & \\
\hline Violence & 0.025 & -0.038 & & & & & & & & & & & & \\
\hline Motion & $-0.301 *$ & -0.083 & 0.119 & & & & & & & & & & & \\
\hline SES & -0.005 & 0.034 & $-0.315^{\star}$ & -0.161 & & & & & & & & & & \\
\hline Cog Stim & -0.117 & -0.013 & 0.003 & 0.223 & $0.388^{* *}$ & & & & & & & & & \\
\hline Cued d' & 0.048 & 0.086 & -0.194 & -0.23 & $0.433^{* * *}$ & 0.122 & & & & & & & & \\
\hline MGA d' & 0.091 & 0.145 & -0.153 & -0.193 & $0.432^{* * *}$ & 0.067 & $0.91^{* * *}$ & & & & & & & \\
\hline $\mathrm{EF}$ & $0.370^{* *}$ & 0.124 & -0.235 & -0.131 & $0.335^{* *}$ & $0.362^{* *}$ & 0.221 & 0.231 & & & & & & \\
\hline Ac Ach & -0.174 & -0.075 & $-0.328^{* * *}$ & 0.094 & $0.333^{* *}$ & 0.245 & 0.117 & 0.073 & $0.254^{*}$ & & & & & \\
\hline $\begin{array}{l}\text { L VVS } \\
\text { Cued }\end{array}$ & $-0.252^{*}$ & 0.183 & -0.154 & 0.056 & $0.264^{*}$ & 0.226 & 0.047 & 0.052 & 0.211 & $0.361 * *$ & & & & \\
\hline $\begin{array}{l}\text { R VVS } \\
\text { Cued }\end{array}$ & -0.194 & 0.223 & -0.199 & 0.099 & $0.273^{*}$ & $0.255^{*}$ & -0.029 & 0 & 0.228 & $0.436^{* * *}$ & $0.871^{* * *}$ & & & \\
\hline $\begin{array}{l}\text { L VVS } \\
\text { MGA }\end{array}$ & $-0.319^{*}$ & 0.197 & -0.053 & 0.072 & 0.137 & 0.129 & 0.01 & 0.057 & 0.123 & $0.281^{*}$ & $0.868^{* * *}$ & $0.701^{* * *}$ & & \\
\hline $\begin{array}{r}\text { R VVS } \\
\text { MGA }\end{array}$ & $-0.264 *$ & $0.271^{*}$ & -0.111 & 0.11 & 0.177 & 0.164 & -0.026 & 0.024 & 0.135 & $0.348^{* *}$ & $0.788^{* * *}$ & $0.86^{* * *}$ & $0.846^{* * *}$ & \\
\hline
\end{tabular}

A

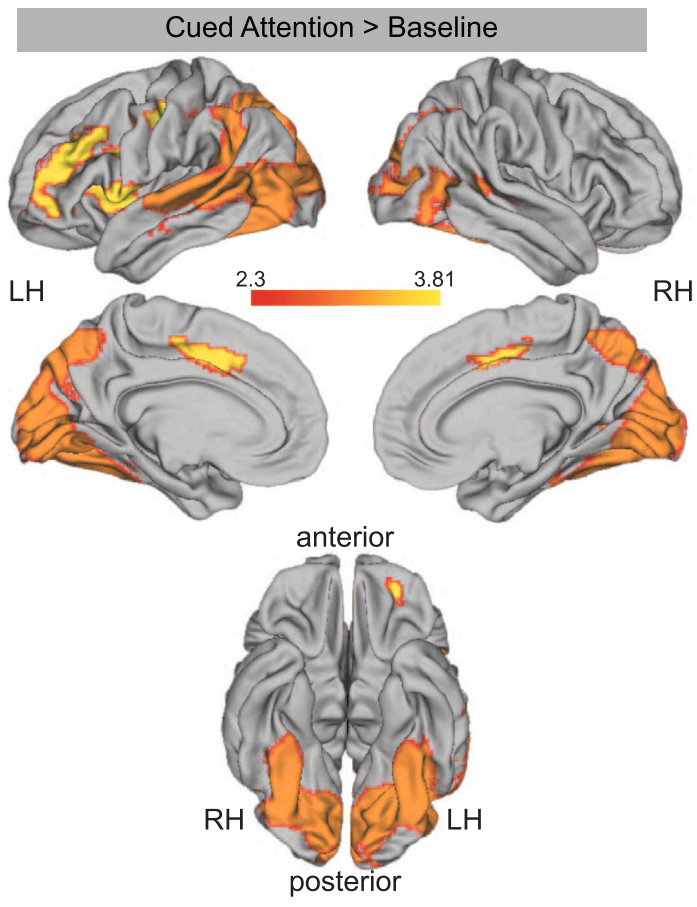

B

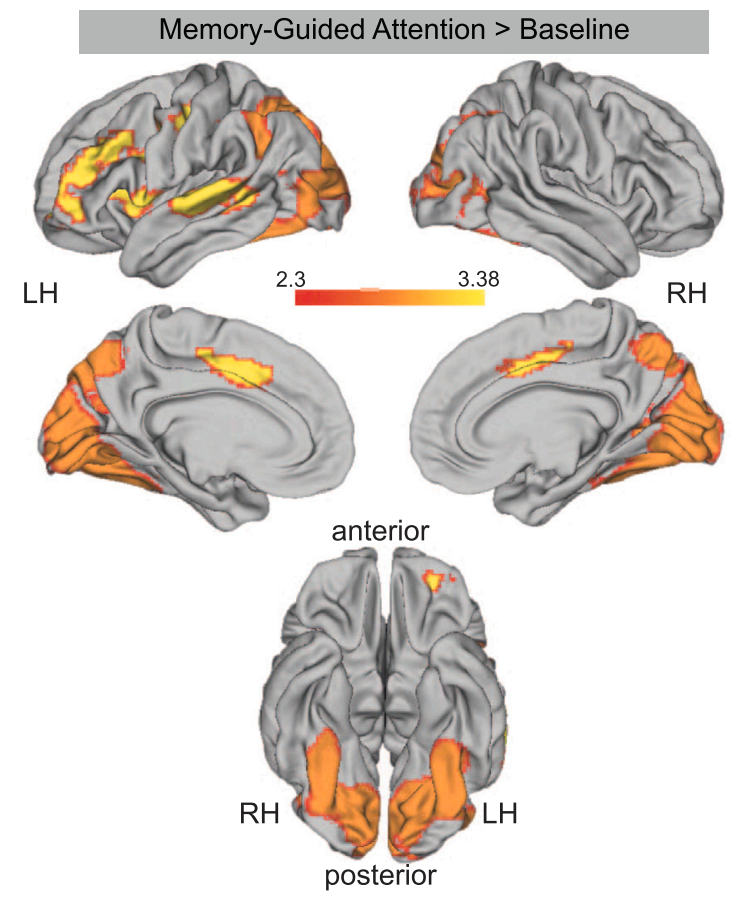

Fig. 4. Whole group, whole brain activation during (A) cued attention $>$ baseline and (B) memory-guided attention $>$ baseline.

attention was significantly associated with $\mathrm{EF}(\beta=0.315, p=.015$; $\beta=0.302, p=.015$, respectively; Fig. 7A). After multiple comparison correction, there was a trend toward a positive association between activation during memory-guided attention and $\mathrm{EF}$ in both left and right VVS ( $\beta=0.255, p=.068 ; \beta=0.237, p=.068$, respectively; Fig. 7B).

\subsection{VVS function, EF, and academic achievement}

We next asked whether VVS function was associated with academic achievement, and whether this association was mediated by differences in EF.

\subsubsection{EF and academic achievement}

As expected, EF was positively associated with academic achievement $(\beta=0.383, p=.005$; Fig. $7 \mathrm{C})$.

\subsubsection{Neural activation and academic achievement}

Next, we examined whether neural activation during cued or memory-guided attention was associated with academic achievement. Whole brain analysis revealed that academic achievement was positively associated with activation during cued attention in two clusters (Fig. 8; Table 3). Consistent with our prediction that VVS function may be related to academic performance, a large cluster spanning left and right ventral temporal cortex was positively associated with academic achievement-this cluster encompassed fusiform gyrus, lingual gyrus, cerebellum, and extended into the right IPS. The second cluster spanned left IPS, cuneus, and lateral occipital cortex and also showed a positive association with academic achievement. 
$\mathbf{A}$

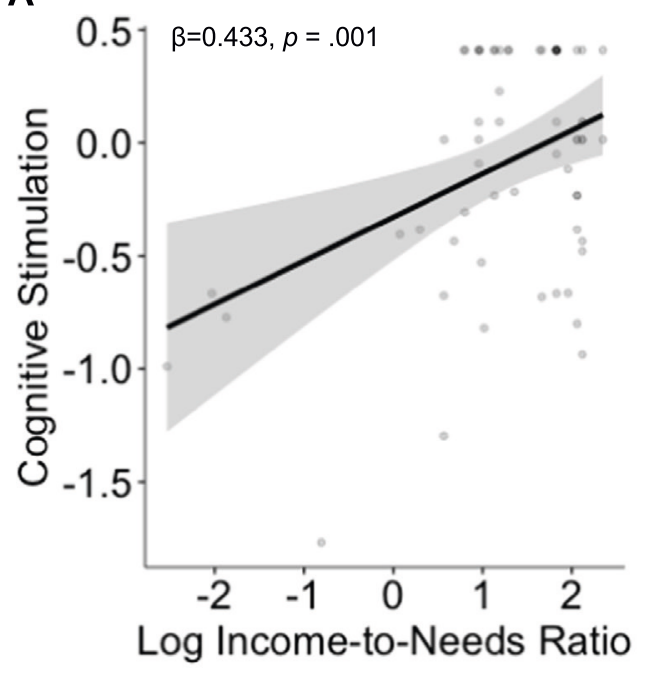

B

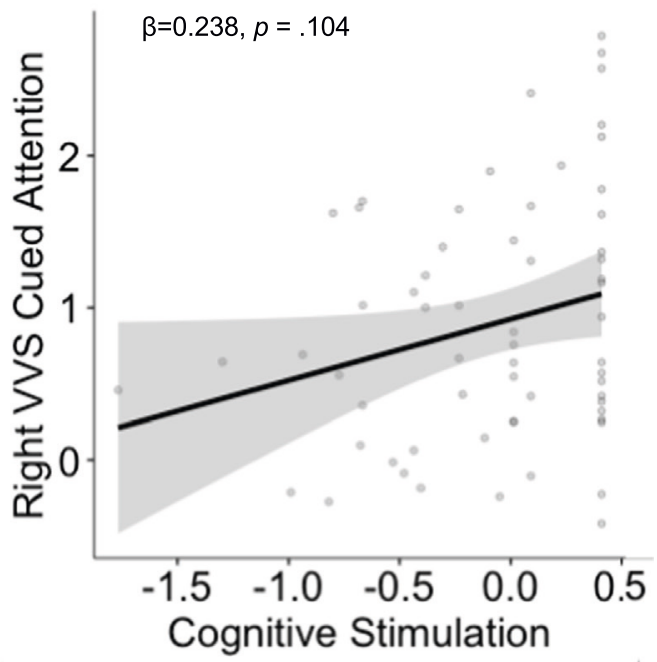

C

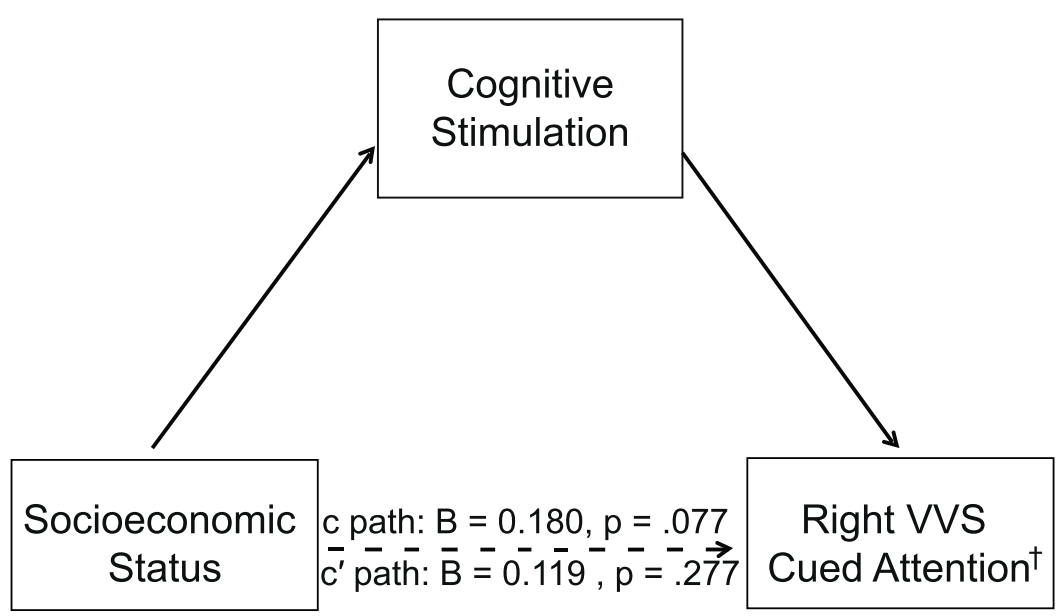

Fig. 5. Cognitive stimulation as a mediator explaining the association between SES and VVS activation. (A) association between INR and cognitive stimulation. (B) Association between cognitive stimulation and right VVS activation during cued attention. (C) Cognitive stimulation marginally mediated the associations between socioeconomic status (log income-to-needs) and right VVS activation during cued attention. Coefficients on scatterplots are standardized. Coefficients on the mediation models are unstandardized. 'indicates marginally significant mediations.

During memory-guided attention, academic achievement was positively associated with one cluster that spanned left and right ventral temporal cortex regions of VVS including the fusiform gyrus and lingual gyrus, and cerebellum (Table 3).

Next, we examined whether activation in VVS ROIs was associated with academic achievement. Consistent with our whole-brain analysis, greater activation in both the left and right VVS was associated with higher academic performance during both cued attention $(\beta=0.367$, $p=.006 ; \beta=0.460, p=.0007$, respectively; Fig. 9B) and memoryguided attention $(\beta=0.281, \quad p=.041 ; \quad \beta=0.378, \quad p=.011$, respectively).

\subsubsection{EF as a mediator of the association between VVS activation and} academic achievement

We next tested the hypothesis that EF mediates the association between VVS activation and academic achievement (Table 4, Fig. 7D\&E). We found a significant indirect effect of left and right VVS activation during cued attention (95\% CI: [.189, 4.159] and 95\% CI: [.132, 3.600], respectively) and of left VVS during memory-guided attention (95\% CI: [.178 4.255] and a trend through activation in the right VVS during memory-guided attention (90\% CI:.152 to 4.061) on academic achievement through EF.

\subsection{INR, VVS function, and academic achievement}

Our final question was whether differences in VVS function mediated the association between INR and academic achievement.

\subsubsection{INR and academic achievement}

Consistent with previous work, INR was positively associated with academic achievement $(\beta=0.258, p=.043)$.

3.6.2. VVS activation as a mediator of the association between INR and academic achievement

Finally, we conducted mediation analyses to determine whether activation in VVS during cued or memory-guided attention mediated the association between INR and academic achievement. We found a significant indirect effect of INR on academic achievement through activation in the right VVS during cued attention (95\% CI: [.088, 2.852], Table 4, Fig. 9C). We additionally found a marginal indirect effect of INR on academic achievement through activation in left VVS during cued attention (90\% CI: [.041, 2.250]). We did not indirect effect of INR on academic achievement through VVS activation during memory-guided attention. 


\section{Income-to-Needs during Cued Attention}

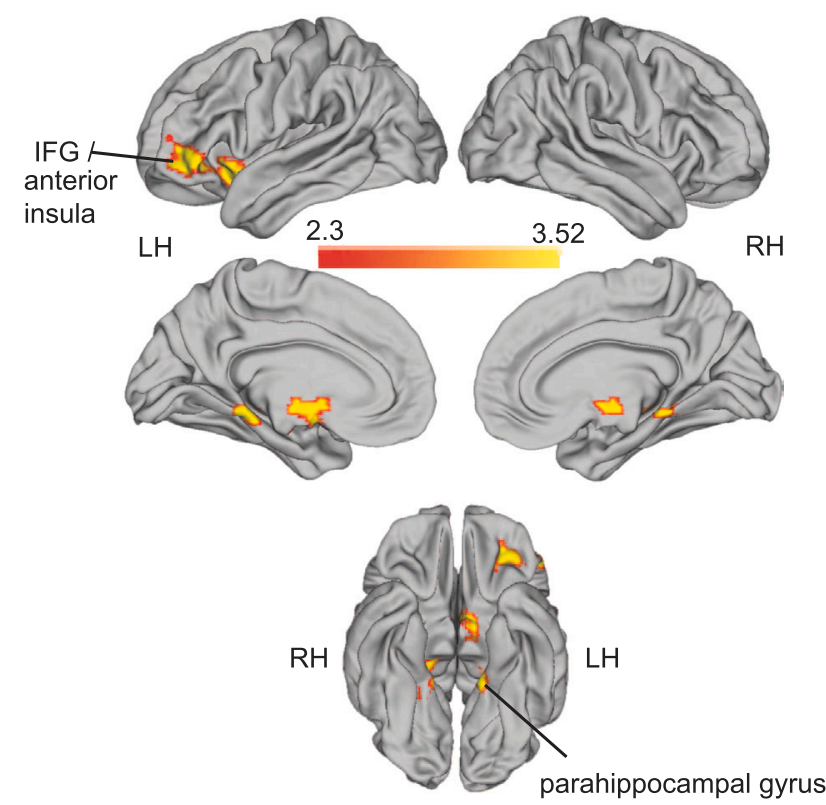

Fig. 6. Whole brain activation associations during cued attention with log income-to-needs as a predictor.

Table 3

Whole brain analyses. Coordinates for the peak intensity are presented in MNI coordinates.

\begin{tabular}{|c|c|c|c|c|c|}
\hline \multicolumn{6}{|l|}{ Cued Attention vs. Baseline } \\
\hline Anatomical Region & $\mathrm{x}$ & $\mathrm{y}$ & $\mathrm{z}$ & voxels & z-max \\
\hline $\begin{array}{l}\text { Bilateral fusiform gyrus, lateral occipital } \\
\text { cortex, intraparietal sulcus, superior } \\
\text { parietal lobule, calcarine sulcus, left } \\
\text { superior temporal sulcus }\end{array}$ & -38 & -66 & -18 & 28479 & 7.9433 \\
\hline $\begin{array}{l}\text { Bilateral dorsal anterior cingulate cortex, } \\
\text { left middle frontal gyrus, anterior } \\
\text { insula, orbitofrontal cortex, precentral } \\
\text { sulcus }\end{array}$ & -4 & 2 & 46 & 5197 & 6.8517 \\
\hline \multicolumn{6}{|l|}{ Memory-Guided Attention vs. Baseline } \\
\hline Anatomical Region & $\mathbf{x}$ & $\mathbf{y}$ & $\mathbf{z}$ & voxels & z-max \\
\hline $\begin{array}{l}\text { Bilateral calcarine sulcus, fusiform gyrus, } \\
\text { lateral occipital cortex, intraparietal } \\
\text { sulcus, superior parietal lobule }\end{array}$ & 8 & -78 & -12 & 21875 & 7.4689 \\
\hline $\begin{array}{l}\text { Dorsal anterior cingulate cortex, left } \\
\text { middle frontal gyrus, anterior insula, } \\
\text { orbitofrontal cortex, precentral sulcus, } \\
\text { superior temporal sulcus }\end{array}$ & -4 & 2 & 46 & 6615 & 7.2078 \\
\hline \multicolumn{6}{|c|}{ Positive Associations with SES during Cued Attention } \\
\hline Anatomical Region & $\mathbf{x}$ & $\mathbf{y}$ & $\mathbf{z}$ & voxels & z-max \\
\hline $\begin{array}{l}\text { Left anterior insula, inferior frontal gyrus, } \\
\text { left putamen, parahippocampal gyrus }\end{array}$ & -34 & 12 & -8 & 1750 & 3.9092 \\
\hline \multicolumn{6}{|c|}{ Positive Associations with Academic Achievement during Cued Attention } \\
\hline Anatomical Region & $\mathbf{x}$ & $\mathbf{y}$ & $\mathbf{z}$ & voxels & z-max \\
\hline $\begin{array}{l}\text { Bilateral cerebellum, fusiform gyrus, } \\
\text { lingual gyrus, right intraparietal sulcus }\end{array}$ & -14 & -66 & -20 & 4919 & 5.3463 \\
\hline $\begin{array}{l}\text { Left intraparietal sulcus, cuneus, lateral } \\
\text { occipital cortex }\end{array}$ & -26 & -84 & 14 & 1412 & 3.655 \\
\hline \multicolumn{6}{|c|}{$\begin{array}{l}\text { Positive Associations with Academic Achievement during Memory-Guided } \\
\text { Attention }\end{array}$} \\
\hline Anatomical Region & $\mathbf{x}$ & $\mathbf{y}$ & $\mathbf{z}$ & voxels & z-max \\
\hline $\begin{array}{l}\text { Bilateral cerebellum, fusiform gyrus, } \\
\text { lingual gyrus }\end{array}$ & -14 & -66 & -20 & 2056 & 5.1195 \\
\hline
\end{tabular}

Table 4

Results from mediation analyses. Confidence intervals not containing 0 indicate evidence for a significantly mediated effect. In some cases, $90 \%$ confidence interval is reported indicating a trend toward a significantly mediated effect. Significant mediations are presented in BOLD and trends are indicated with a *.

\begin{tabular}{|c|c|}
\hline \multicolumn{2}{|c|}{$\begin{array}{l}\text { Cognitive Stimulation as a mediator of the association between SES and VVS } \\
\text { activation }\end{array}$} \\
\hline & 95\% Confidence Interval \\
\hline Left VVS Cued & {$[-.047, .152]$} \\
\hline Right VVS Cued & {$[-.010, .183]\left(90 \%\right.$ CI: $\left.[.002, .160]^{*}\right)$} \\
\hline Left VVS MGA & {$[-.047, .155]$} \\
\hline Right VVS MGA & {$[-.051, .134]$} \\
\hline \multicolumn{2}{|c|}{$\begin{array}{l}\mathrm{EF} \text { as a mediator of the association between VVS activation and Academic } \\
\text { Achievement }\end{array}$} \\
\hline & 95\% Confidence Interval \\
\hline Left VVS Cued & {$[.189,4.159]$} \\
\hline Right VVS Cued & [.132 to 3.600$]$ \\
\hline Left VVS MGA & {$[.178,4.255]$} \\
\hline Right VVS MGA & $-.007,4.764]$ (90\% CI: $\left.[.152,4.061]^{*}\right)$ \\
\hline \multicolumn{2}{|c|}{$\begin{array}{l}\text { VVS activation as a mediator of the association between SES and Academi } \\
\text { Achievement }\end{array}$} \\
\hline & 95\% Confidence Interval \\
\hline Left VVS Cued & {$[-.048, .148]\left(90 \%\right.$ CI: $\left.[.041 \text { to } 2.250]^{*}\right)$} \\
\hline Right VVS Cued & [.088 to 2.852$]$ \\
\hline Left VVS MGA & {$[-.231,1.692]$} \\
\hline Right VVS MGA & {$[-.183,1.951]$} \\
\hline
\end{tabular}

\section{Discussion}

The present study adds to the growing body of literature suggesting that SES-related differences in neural function may contribute to the income-achievement gap. Prior models of neurodevelopmental mechanisms of the achievement gap have chiefly focused on networks involved in complex cognitive functions. While these higher-order cognitive regions clearly play a role in the income-achievement gap, here, we provide novel evidence that neural function in earlier-developing visual processing regions may also play a role in these disparities and provide some initial evidence that cognitive stimulation may be important for VVS function. Specifically, (i) childhood SES was marginally associated with activation in VVS during cued attention, (ii) cognitive stimulation marginally explained the association between SES and VVS activation during cued attention; (iii) VVS activation during both cued and memory-guided attention predicted children's academic achievement, (iv) the link between VVS activation during both tasks and academic achievement was mediated by differences in EF, and (v) VVS recruitment during cued attention mediated the association between SES and achievement. These findings extend prior work by demonstrating not only that childhood SES is associated with the functional activation in visual processing regions, but also that cognitive stimulation could contribute to these differences in the function. Moreover, these differences in visual processing may contribute to higher-order cognitive function, which in turn may play a role in explaining individual differences in academic achievement. We acknowledge that several of the hypothesized associations were marginal and should be interpreted with caution until replicated.

\subsection{SES, cognitive stimulation, and VVS function}

Why might childhood SES be associated with development of VVS function? The ventral temporal cortex contains numerous brain regions that respond preferentially to particular categories of stimuli, such as scenes, faces, and objects (Ungerleider and Haxby, 1994). Infants already demonstrate category-preferential responses in VVS (Deen et al., 2017), but it is possible that environmental experience influences how strong these category preferences are or how effectively these regions maintain representations during a cognitively-demanding task. SES has been consistently associated with structure and function of VVS (Finn et al., 2017; Leonard et al., 2019; Mackey et al., 2015; Noble et al., 2015; 
A

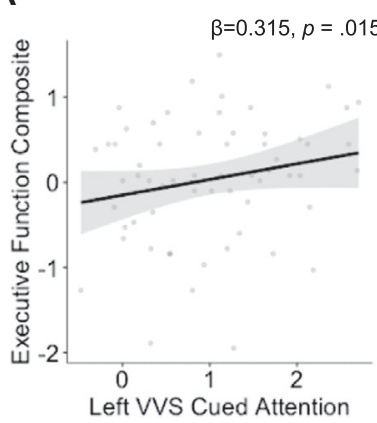

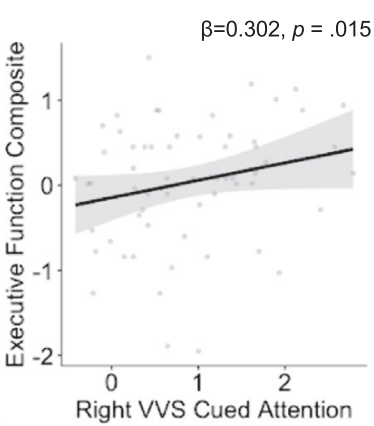

B

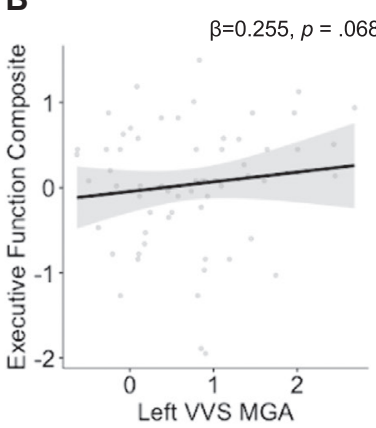

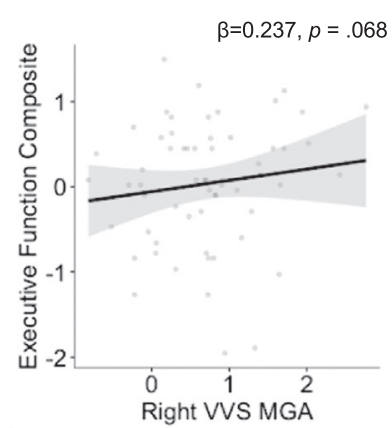
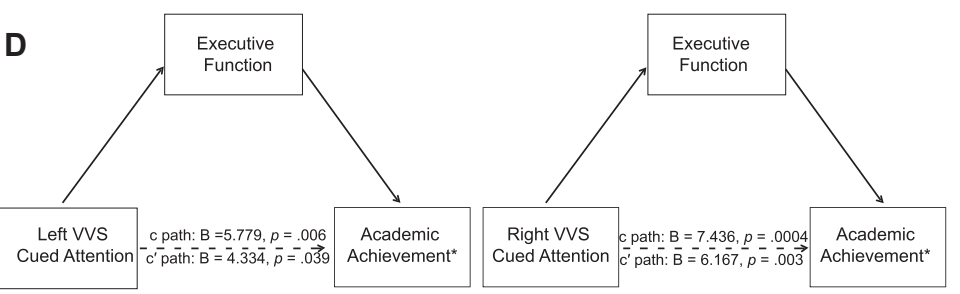

C

$\beta=0.383, p=.005$

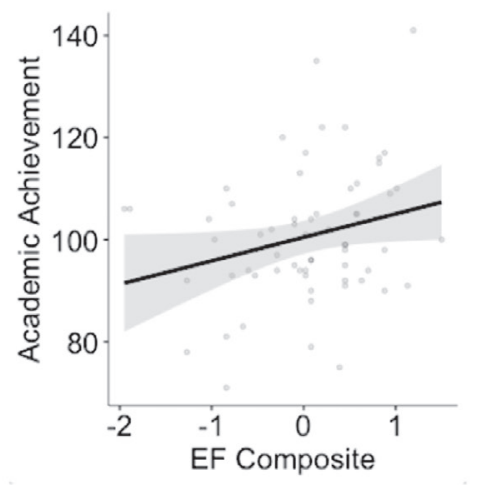

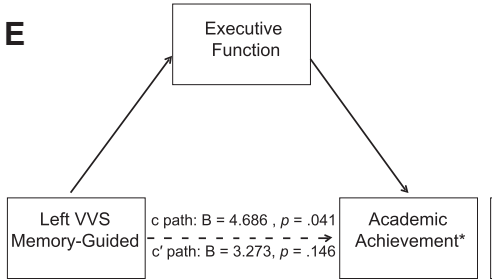

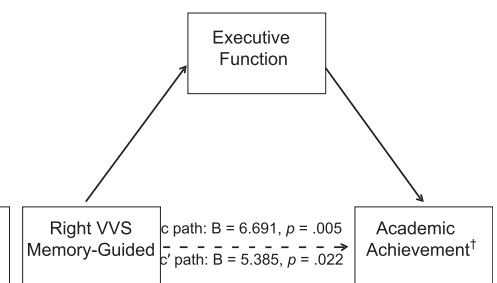

Fig. 7. Executive function (EF) as a mediator explaining ventral visual stream (VVS) association with academic achievement. (A) associations between activation in VVS during cued attention and EF. (B) Associations between activation during memory-guided attention (MGA) and EF. (C) Associations between EF and academic achievement. EF significantly mediated the associations between VVS activation academic achievement during cued attention (D) and memory-guided attention (E). Coefficients on scatterplots are standardized. Coefficients on the mediation models are unstandardized. 'indicates marginally significant mediations. *indicates statistically significant mediations.

\section{Academic Achievement during Cued Attention}

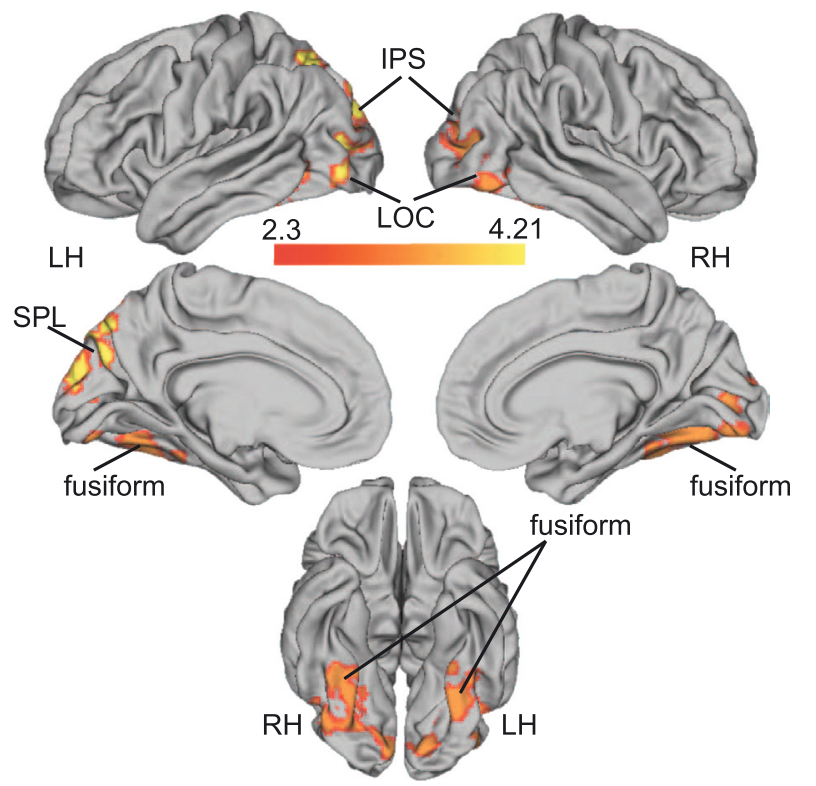

Fig. 8. Whole brain activation associations with academic achievement during cued attention.
Rosen et al., 2018; Ursache and Noble, 2016). We recently proposed that SES-related differences in structure and function of VVS may be traceable, at least in part, to a malleable aspects of the early environment-the degree of cognitive stimulation occurring in the context of caregiver interactions (Rosen, Amso, et al., 2019). Consistent with our hypothesis, we found that cognitive stimulation including a rich linguistic environment and high caregiver involvement in learning was associated marginally with greater recruitment of VVS during cued attention. Caregiver-child interactions that encourage children to maintain attention and highlight features of different stimuli in the environment could help children form semantic representations that contribute to the development of VVS. Moreover, we found that cognitive stimulation marginally explained SES-related differences in recruitment of VVS during cued attention, highlighting how cognitive stimulation may play an important role in SES-related differences in VVS function. While these findings are consistent with our hypothesis that caregiver-mediated cognitive stimulation is important for VVS development, the results were marginal after multiple comparison correction and should be interpreted with caution. Future studies should aim to replicate these results in a larger sample.

\subsection{SES, VVS function, EF, and academic achievement}

We found that differences in VVS function were associated with academic achievement and mediated the association between SES and academic achievement. Why might VVS function contribute to differences in academic outcomes? Our model and others posit that development of VVS scaffolds development of the PFC, which in turn may influence development of higher-order cognitive skills and academic 
A

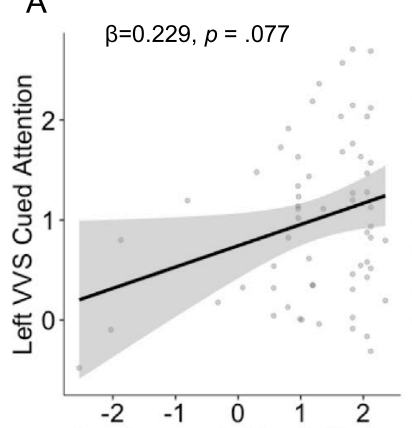

Log Income to Needs Ratio

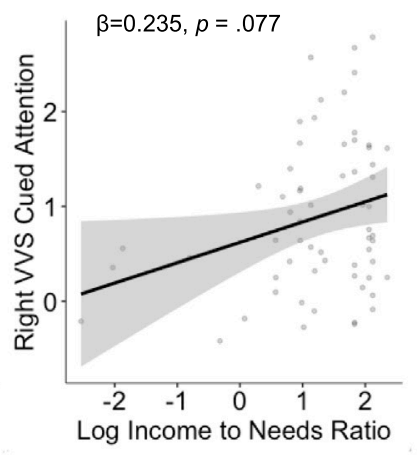

Log Income to Needs Ratio
B

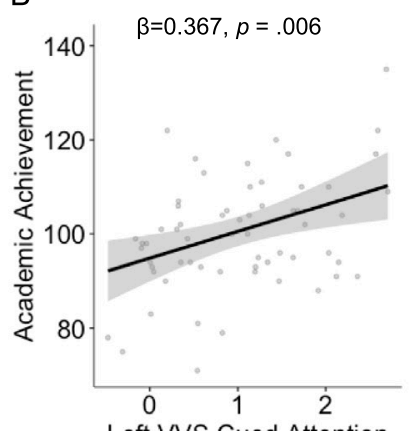

Left VVS Cued Attention

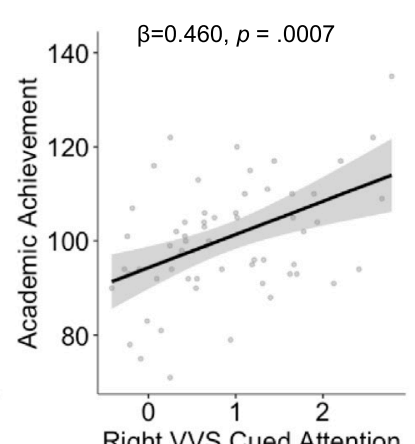

Right VVS Cued Attention
C

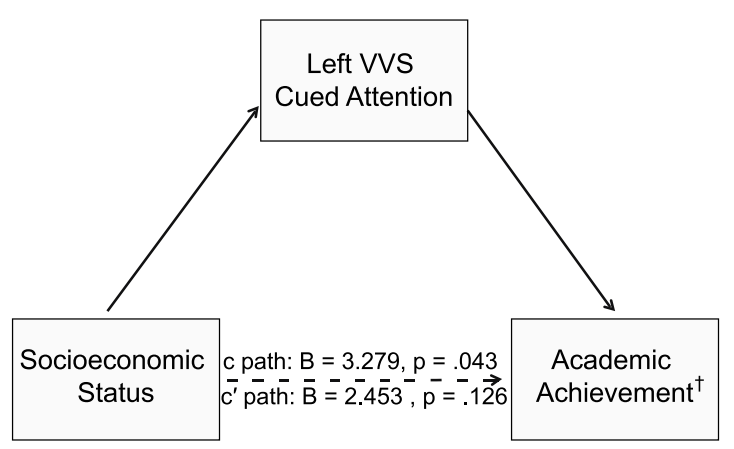

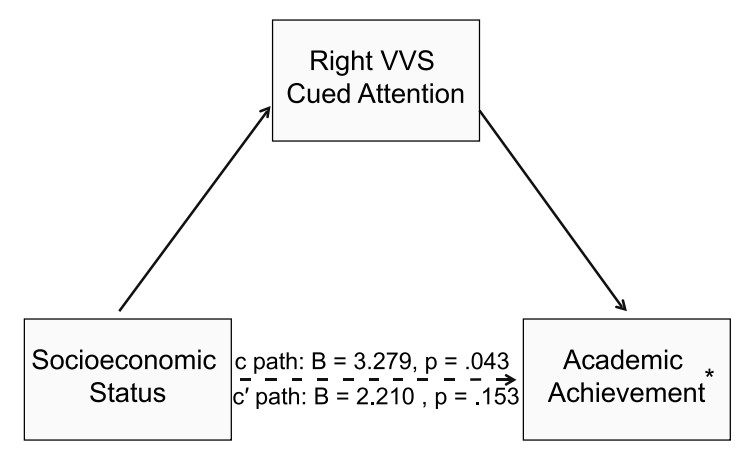

Fig. 9. VVS activation as a mediator in the association between SES and academic achievement. (A) associations between INR and activation in VVS during cued attention. (B) associations between activation in VVS during cued attention and academic achievement. (C) Activation in the VVS during cued attention mediated the association between socioeconomic status (log income-to-needs) and academic achievement. Coefficients on scatterplots are standardized. Coefficients on the mediation models are unstandardized. * indicates significant mediations. ${ }^{\dagger}$ indicates marginally significant mediations.

achievement (Amso and Scerif, 2015; Rosen et al., 2019). Indeed, here we found that VVS activation was associated with EF performance and individual differences in EF mediated the association of VVS activation with academic achievement. Limited one-on-one caregiver interactions and reduced access to complex stimuli may constrain the amount of organized feed-forward information that is sent from VVS to regions of the brain that support higher-order cognitive function. Over development, this may result in differences higher-order cognitive functioning. Several previous studies have highlighted that development of VVS partially explains age-related increases in higher-order cognitive performance including working memory and long-term memory (Chai et al., 2010; Rosen et al., 2018; Wendelken et al., 2011). Our findings that VVS recruitment mediates the association between SES and academic achievement is consistent with a recent study demonstrating that reduced recruitment of the fusiform gyrus during a working memory task for faces was associated with reduced task performance and mediated SES-related reductions in academic achievement (Rosen et al., 2018). Taken together, these findings suggest that these regions may play an important role in the development of higher-order cognition and that alterations in VVS development among children raised in low-SES environments could have downstream impacts on cognitive development and, in turn, on academic achievement.

\subsection{Under what circumstances do SES-related differences in VVS emerge?}

To date, studies showing that SES-related differences in function of VVS have used tests of attention and working memory (Finn et al., 2017; Rosen et al., 2018). Therefore, we do not yet know whether SES-related differences in the function of VVS emerge only during cognitively demanding tasks or whether these differences are also present during basic visual processing. Given that several studies have observed structural differences in VVS as a function of SES (Leonard et al., 2019; Mackey et al., 2015; Noble et al., 2015; Piccolo et al., 2016), it is possible that the differences seen during attention and memory tasks reflect differences in more basic aspects of visual processing. Alternatively, it is possible that basic visual processing of these regions is similar among children across the SES distribution, and that the ability to maintain attention on representations in VVS is influenced more specifically by SES. If so, it could be that top-down modulation of VVS during cognitively demanding tasks that require attention or holding visual information in working memory (Gazzaley and Nobre, 2012) may be reduced in children from lower SES backgrounds. Future studies should include both a VVS localizer task using a variety of different categories of visual stimuli (e.g. faces, scenes, objects) as well as attention or working memory tasks with these different stimulus categories to disentangle whether differences emerge during basic visual processing or only during cognitively demanding tasks.

Interestingly, we only found an association between SES and recruitment of VVS during cued but not memory-guided attention. VVS recruitment is generally higher during cued compared to memoryguided attention (Rosen et al., 2016; Rosen et al., 2018). Indeed, in the present study of young children we saw greater recruitment of VVS during cued compared to memory-guided attention across the whole sample. Therefore, SES-related differences may emerge especially during tasks that rely heavily on VVS.

\subsection{SES-related differences in frontoparietal control network}

The frontoparietal control network has been the focus of prior work on the achievement gap because of the important role that it plays in the top-down goal-directed behavior necessary for academic performance 
(Finn et al., 2017; Rosen et al., 2018). Indeed, many previous studies have found SES-related differences in structure, connectivity, and activation of the lateral prefrontal cortex for attention, executive functions and language tasks (Farah, 2017; Finn et al., 2016; Noble et al., 2015; Raizada et al., 2008; Sheridan et al., 2012; Tooley et al., 2019). Consistent with previous findings, we also found that SES was positively associated with recruitment of the right inferior frontal gyrus and anterior insula during cued attention, regions that have been found to play an important role in focused attention, attentional orienting, and cognitive control (Doricchi et al., 2010; Hampshire et al., 2010; Jiang et al., 2015; Wu et al., 2019). These findings add to the well-documented results that SES is associated with recruitment of prefrontal cortex regions in children during cognitively demanding tasks.

\subsection{Limitations}

The present study has several limitations that should be acknowledged. First, while the narrow age range allowed us to investigate the neural processes underlying cued and memory-guided attention and their relation to academic achievement, we are limited in our ability to understand the neurodevelopmental trajectory of cued and memoryguided attention (see Supplemental materials for additional discussion). Future studies with large samples that span early childhood to adulthood would help to elucidate at what age "adult-like" processing emerges, particularly for memory-guided compared to cued attention. Relatedly, given that the present study only had one neuroimaging time point, we are not able to assess the timing of development of VVS. Future longitudinal studies starting early in development will be needed to determine whether SES-related differences in neural structure and function of VVS precedes and predicts SES-related differences in prefrontal cortex development and downstream impacts on cognitive and academic outcomes.

This study did not include a passive viewing condition. Studies in adults have used the cued attention task as a baseline to investigate recruitment specific to memory-guided attention over and above a more traditional attention task (Rosen et al., 2016; Rosen et al., 2018; Summerfield et al., 2006). However, neither task serves as an appropriate baseline condition for the other when considering the associations between SES and neural activity. That is to say that we do not see a reason to expect that SES would be strongly associated with activity in one task compared to the other (e.g. cued attention $>$ memory-guided attention) and therefore using one as a baseline for the other may obscure any true associations between SES and neural activation. As such, we used fixation blocks as a baseline condition for each when looking at the associations with SES, home environment, and achievement. A passive condition that still contained visual stimuli but in which participants were asked to simply look at the screen without engaging in any attentionally demanding task would have provided a better baseline to ensure that the differences seen are due to the task and not the stimuli.

It would be especially interesting to know whether our reported results extend to other sensory modalities. One study found reductions in somatosensory processing in children from lower-SES backgrounds, as compared to children from higher-SES backgrounds (Weiss et al., 2018). Future studies should employ attentional tasks across several modalities (auditory, visual, tactile) to determine whether SES-related differences emerge in sensory processing regions for multiple modalities and whether these differences drive academic outcomes.

Additionally, while healthy amounts of cognitive stimulation may be important for the development of the VVS, EF, and academic achievement, a recent study suggests that mothers who were randomly assigned to teach their child something not only increased their cognitive stimulation but also increased intrusiveness (King et al., Under Review). Recent work also demonstrates that high greater parental intrusiveness is linked to lower self-regulation and performance on EF tasks in children (Obradivic et al., 2021). Therefore, future work should focus on a more nuanced understanding of the circumstances under which cognitive stimulation is beneficial for cognitive and neural development.

We acknowledge that many of the findings in this study were marginal and should be interpreted with caution. We hope this work with spark larger studies to further investigate the role of VVS in the incomeachievement gap as well as whether cognitive stimulation drives these differences.

Finally, we made attempts to recruit a racially and ethnically diverse sample from across the income distribution, but ultimately the population recruited reflected the demographics of the Seattle area and thus was predominately white. Additionally, the income distribution of the present sample broadly covered poverty to affluence with most families living well above the poverty line. As such, the constraints on the generality of the present study should be considered (Simons et al., 2017). Future studies should determine the generalization of these findings to a more racially and ethnically diverse population of children as well as be replicated in other geographical regions and among samples including more children living in poverty.

\subsection{Conclusions}

The present study adds to a growing body of literature that highlights the important role of VVS in SES-related differences in academic outcomes. Most previous work on this topic has focused on the frontoparietal network that is involved in top-down goal-directed attention. Our findings extend prior research by demonstrating that (i) VVS activation differs as a function of childhood SES, (ii) is associated with executive functions and academic outcomes, and (iii) serves as an additional neurodevelopmental mechanism contributing to the incomeachievement gap. Furthermore, this work highlights a potential role that cognitive stimulation plays in SES-related differences in VVS function, highlighting a potential environmental mechanism that may be contributing to SES-related differences in both neural function and academic outcomes.

\section{Declaration of Competing Interest}

The authors declare that they have no known competing financial interests or personal relationships that could have appeared to influence the work reported in this paper.

\section{Data Availability}

Raw data for this project are available on the Open Science Framework (https://osf.io/s78aq/).

\section{Acknowledgements}

The authors would like to thank Kudzaiishe (Irene) Mapfunde for her assistance with data collection, Nessa Bryce for creating the illustration in Fig. 1, and Rachel Romeo for her thoughtful comments. This work was supported by the National Institute of Child Health and Human Development at the National Institute of Health (F32-HD089514 and K99HD099203 to M.L.R), the National Institute of Mental Health at the National Institute of Health, U.S.A. (R01-MH103291, R01-MH106482, R56-MH119194, and R37-MH119194 to K.A.M) and the Institute for Learning \& Brain Sciences (University of Washington, Seattle, U.S.A) Ready Mind Project to A.N.M.

\section{Appendix A. Supporting information}

Supplementary data associated with this article can be found in the online version at doi:10.1016/j.dcn.2021.101025. 


\section{References}

Amso, D., Scerif, G., 2015. The attentive brain: insights from developmental cognitive neuroscience. Nat. Rev. Neurosci. 16 (10), 606-619. https://doi.org/10.1038/ nrn4025.

Blair, C., Razza, R.P., 2007. Relating effortful control, executive function, and false belief understanding to emerging math and literacy ability in kindergarten. Child Dev. 78 (2), 647-663. https://doi.org/10.1111/j.1467-8624.2007.01019.x.

Bradley, R.H., Corwyn, R.F., McAdoo, H.P., Garcia Coll, C., 2001. The home environments of children in the United States Part I: variations by age, ethnicity, and poverty status. Child Dev. 72 (6), 1844-1867. https://doi.org/10.1111/1467-8624. t01-1-00382.

Brooks, R., Singleton, J.L., Meltzoff, A.N., 2019. Enhanced gaze-following behavior in deaf infants of deaf parents. Dev. Sci. 23, e12900 https://doi.org/10.1111/ desc. 12900.

Carlson, S.M., Meltzoff, A.N., 2008. Bilingual experience and executive functioning in young children. Dev. Sci. 11 (2), 282-298. https://doi.org/10.1111/j.1467. 7687.2008.00675.x.

Chai, X.J., Ofen, N., Jacobs, L.F., Gabrieli, J.D.E., 2010. Scene complexity: influence on perception, memory, and development in the medial temporal lobe. Front. Hum. Neurosci. 4, 21. https://doi.org/10.3389/fnhum.2010.00021.

Deen, B., Richardson, H., Dilks, D.D., Takahashi, A., Keil, B., Wald, L.L., Kanwisher, N., Saxe, R., 2017. Organization of high-level visual cortex in human infants. Nat. Commun. 8, 13995. https://doi.org/10.1038/ncomms13995.

Dixon, M.L., Zelazo, P.D., De Rosa, E., 2010. Evidence for intact memory-guided attention in school-aged children. Dev. Sci. 13 (1), 161-169. https://doi.org/ 10.1111/j.1467-7687.2009.00875.x.

Doricchi, F., MacCi, E., Silvetti, M., MacAluso, E., 2010. Neural correlates of the spatial and expectancy components of endogenous and stimulus-driven orienting of attention in the posner task. Cereb. Cortex 20 (7), 1574-1585. https://doi.org/ 10.1093/cercor/bhp215.

Eklund, A., Nichols, T.E., Knutsson, H., 2016. Cluster failure: why fMRI inferences for spatial extent have inflated false-positive rates. Proc. Natl. Acad. Sci. 113 (28), 7900-7905. https://doi.org/10.1073/pnas.1602413113.

Farah, M.J., 2017. The neuroscience of socioeconomic status: correlates, causes, and consequences. Neuron 96 (1), 56-71. https://doi.org/10.1016/j. neuron.2017.08.034.

Finn, A.S., Minas, J.E., Leonard, J.A., Mackey, A.P., Salvatore, J., Goetz, C., West, M.R., Gabrieli, C.F.O., Gabrieli, J.D.E., 2017. Functional brain organization of working memory in adolescents varies in relation to family income and academic achievement. Dev. Sci. 20 (5) https://doi.org/10.1111/desc.12450.

Fox, N.A., Leavitt, L.A., 1995. The Violence Exposure Scale for Children-Revised ((VEX$\mathrm{R})$ ).

Gazzaley, A., Nobre, A.C., 2012. Top-down modulation: bridging selective attention and working memory. Trends Cogn. Sci. 16 (2), 129-135. https://doi.org/10.1016/j. tics.2011.11.014.

Hampshire, A., Chamberlain, S.R., Monti, M.M., Duncan, J., Owen, A.M., 2010. The role of the right inferior frontal gyrus: inhibition and attentional control. NeuroImage 50 (3), 1313-1319. https://doi.org/10.1016/j.neuroimage.2009.12.109.

Hanushek, E.A., Peterson, P.E., Talpey, L.M., Woessmann, L., 2019. The achievement gap fails to close. EducationNext.

Hayes, A., 2013. Introduction to Mediation, Moderation, and Conditional Process Analysis. Guilford, New York, NY, pp. 3-4 https://doi.org/978-1-60918-230-4.

Hayes, A.F., 2009. Beyond Baron and Kenny: statistical mediation analysis in the new millennium. Commun. Monogr. 76, 408-420. https://doi.org/10.1080/ 03637750903310360.

Jiang, J., Beck, J., Heller, K., Egner, T., 2015. An insula-frontostriatal network mediates flexible cognitive control by adaptively predicting changing control demands. Nat. Commun. 6, 8165. https://doi.org/10.1038/ncomms9165.

King, L., Range, E., Gotlib, I., Humphreys, K. (Under Review). Teaching or learning form baby: Inducing explicit parenting goals influences caregiver intrusiveness. hhttps ://psyarxiv.com/g9rvn/ $/$.

Lawson, G.M., Hook, C.J., Farah, M.J., 2018. A meta-analysis of the relationship between socioeconomic status and executive function performance among children. Dev. Sci. 21 (2) https://doi.org/10.1111/desc.12529.

Leonard, J.A., Romeo, R.R., Park, A.T., Takada, M.E., Robinson, S.T., Grotzinger, H., Last, B.S., Finn, A.S., Gabrieli, J.D.E., Mackey, A.P., 2019. Associations between cortical thickness and reasoning differ by socioeconomic status in development. Dev. Cogn. Neurosci. 36, 100641 https://doi.org/10.1016/j.dcn.2019.100641.

Lipina, S., Segretin, S., Hermida, J., Prats, L., Fracchia, C., Camelo, J.L., Colombo, J., 2013. Linking childhood poverty and cognition: environmental mediators of nonverbal executive control in an Argentine sample. Dev. Sci. 16 (5), 697-707. https:// doi.org/10.1111/desc. 12080 .

Lurie, L.A., Hagen, M.P., McLaughlin, K.A., Sheridan, M.A., Meltzoff, A.N., Rosen, M.L. 2021. Mechanisms linking socioeconomic status and academic achievement: cognitive stimulation and language. Cogn. Dev. 58, 101045 https://doi.org/ 10.10.1016/j.cogdev.2021.101045.

Mackey, A.P., Finn, A.S., Leonard, J.A., Jacoby-Senghor, D.S., West, M.R., Gabrieli, C.F. O., Gabrieli, J.D.E., 2015. Neuroanatomical correlates of the income-achievement gap. Psychol. Sci. 26 (6), 925-933. https://doi.org/10.1177/0956797615572233.

MacKinnon, D.P., Fairchild, A.J., Fritz, M.S., 2007. Mediation analysis. Ann. Rev. Psychol. 58, 593-602. https://doi.org/10.1146/annurev.psych.58.110405.085542. Mediation.

MacKinnon, D.P., Krull, J.L., Lockwood, C.M., 2000. Equivalence of the mediation, confounding and suppression effect. Prev. Sci. 1 (4), 173-181. https://doi.org/ 10.1023/A:1026595011371.
McDermott, C.L., Seidlitz, J., Nadig, A., Liu, S., Clasen, L.S., Blumenthal, J.D., Reardon, P.K., Lalonde, F., Greenstein, D., Patel, R., Chakravarty, M.M., Lerch, J.P., Raznahan, A., 2019. Longitudinally mapping childhood socioeconomic status associations with cortical and subcortical morphology. J. Neurosci. 39 (8), 1365-1373. https://doi.org/10.1523/JNEUROSCI.1808-18.2018.

McLaughlin, K.A., Green, J.G., Gruber, M.J., Sampson, N.A., Zaslavsky, A.M., Kessler, R. C., 2012. Childhood adversities and first onset of psychiatric disorders in a national sample of US adolescents. Arch. Gen. Psychiatry 69 (11), 1151-1160. https://doi. org/10.1001/archgenpsychiatry.2011.2277.

Mezzacappa, E., 2004. Alerting, orienting, and executive attention: developmental properties and sociodemographic correlates in an epidemiological sample of young, urban children. Child Dev. 75 (5), 1373-1386. https://doi.org/10.1117/12.572040.

Noble, K.G., Houston, S.M., Brito, N.H., Bartsch, H., Kan, E., Kuperman, J.M., Akshoomoff, N., Amaral, D.G., Bloss, C.S., Libiger, O., Schork, N.J., Murray, S.S., Casey, B.J., Chang, L., Ernst, T.M., Frazier, J.A., Gruen, J.R., Kennedy, D.N., Van Zijl, P., Sowell, E.R., 2015. Family income, parental education and brain structure in children and adolescents. Nat. Neurosci. 18 (5), 773-778. https://doi.org/10.1038/ nn.3983.

Noble, K.G., McCandliss, B.D., Farah, M.J., 2007. Socioeconomic gradients predict individual differences in neurocognitive abilities. Dev. Sci. 10 (4), 464-480. https:// doi.org/10.1111/j.1467-7687.2007.00600.x.

Noble, K.G., Norman, M.F., Farah, M.J., 2005. Neurocognitive correlates of socioeconomic status in kindergarten children. Dev. Sci. 8 (1), 74-87. https://doi. org/10.1111/j.1467-7687.2005.00394.x.

Nussenbaum, K., Scerif, G., Nobre, A.C., 2019. Differential effects of salient visual events on memory-guided attention in adults and children. Child Dev. 90 (4), 1369-1388. https://doi.org/10.1111/cdev.13149.

Obradivic, J., Sulik, M.J., Shaffer, A., 2021. Learning to let go: parental over-engagement predicts poorer self-regulation in kindergarteners. J. Family Psychol. https://doi. org/10.1037/fam0000838.

Perchet, C., García-Larrea, L., 2000. Visuospatial attention and motor reaction in children: an electrophysiological study of the "Posner" paradigm. Psychophysiology 37 (2), 231-241. https://doi.org/10.1017/S0048577200981368.

Piccolo, L.R., Merz, E.C., He, X., Sowell, E.R., Noble, K.G., 2016. Age-related differences in cortical thickness vary by socioeconomic status. PLoS One 11 (9), 0162511. https://doi.org/10.1371/journal.pone.0162511.

Rabiner, D.L., Carrig, M.M., Dodge, K.A., 2016. Attention problems and academic achievement: do persistent and earlier-emerging problems have more adverse longterm effects? J. Atten. Disord. 20 (11), 946-957. https://doi.org/10.1177/ 1087054713507974 .

Rabiner, D.L., Murray, D.W., Schmid, L., Malone, P.S., 2004. An exploration of the relationship between ethnicity, attention problems, and academic achievement. School Psychol. Rev. 33 (4), 498-509.

Raizada, R.D.S., Richards, T.L., Meltzoff, A., Kuhl, P.K., 2008. Socioeconomic status predicts hemispheric specialisation of the left inferior frontal gyrus in young children. NeuroImage 40, 1392-1401. https://doi.org/10.1016/j. neuroimage.2008.01.021.

Reardon, S.F., 2013. The widening income achievement gap. Educ. Leadersh.

Romeo, R.R., Leonard, J.A., Robinson, S.T., West, M.R., Mackey, A.P., Rowe, M.L., Gabrieli, J.D.E., 2018. Beyond the 30-million-word gap: children's conversational exposure is associated with language-related brain function. Psychol. Sci. 29 (5), 700-710. https://doi.org/10.1177/0956797617742725.

Rosen, M.L., Amso, D., McLaughlin, K.A., 2019. The role of the ventral visual stream in scaffolding prefrontal cortex development: a novel mechanism linking socioeconomic status and executive function. Dev. Cogn. Neurosci. 39, 100699 https://doi.org/10.1016/j.dcn.2019.100699.

Rosen, M.L., Hagen, M.P., Lurie, L.A., Miles, Z.E., Sheridan, M.A., Meltzoff, A.N., McLaughlin, K.A., 2020. Cognitive stimulation as a mechanism linking socioeconomic status with executive Ffunction: s longitudinal investigation. Child Dev. 91 (4), e762-e779. https://doi.org/10.1111/cdev.13315.

Rosen, M.L., Meltzoff, A.N., Sheridan, M.A., McLaughlin, K.A., 2019. Distinct aspects of the early environment contribute to associative memory, cued attention, and memory-guided attention: implications for academic achievement. Dev. Cogn. Neurosci. 40, 100731 https://doi.org/10.1016/j.dcn.2019.100731.

Rosen, M.L., Sheridan, M.A., Sambrook, K.A., Meltzoff, A.N., McLaughlin, K.A., 2018. Socioeconomic disparities in academic achievement: a multi-modal investigation of neural mechanisms in children and adolescents. NeuroImage 173, 298-310. https:// doi.org/10.1016/j.neuroimage.2018.02.043.

Rosen, M.L., Sheridan, M.A., Sambrook, K.A., Peverill, M.R., Meltzoff, A.N., McLaughlin, K.A., 2018. The role of visual association cortex in associative memory formation across development. J. Cogn. Neurosci. 30 (3), 365-380. https://doi.org/ $10.1162 /$ jocn a 01202.

Rosen, M.L., Stern, C.E., Devaney, K.J., Somers, D.C., 2018. Cortical and subcortical contributions to long-term memory-guided visuospatial attention. Cereb. Cortex 28 (8), 2935-2947. https://doi.org/10.1093/cercor/bhx172.

Rosen, M.L., Stern, C.E., Michalka, S.W., Devaney, K.J., Somers, D.C., 2016. Cognitive control network contributions to memory-guided visual attention. Cereb. Cortex 26 (5), 2059-2073. https://doi.org/10.1093/cercor/bhv028.

Rothbart, M.K., Sheese, B.E., Posner, M.I., 2007. Executive attention and effortful control: linking temperament, brain networks, and genes. Child Dev. Perspect. 1 (1), 2-7. https://doi.org/10.1111/j.1750-8606.2007.00002.x.

Rowe, M.L., 2008. Child-directed speech: relation to socioeconomic status, knowledge of child development and child vocabulary skill. J. Child Lang. 35 (1), 185-205. https://doi.org/10.1017/S0305000907008343. 
Samuels, W.E., Tournaki, N., Blackman, S., Zilinski, C., 2016. Executive functioning predicts academic achievement in middle school: a four-year longitudinal study. J. Educ. Res. 109 (5), 478-490. https://doi.org/10.1080/00220671.2014.979913.

Schrank, F.A., Mather, N., McGrew, K.S., 2015. Test review: Woodcock-Johnson IV tests of achievement. J. Psychoeduc. Assess. https://doi.org/10.1177/ 0734282915569447.

Senju, A., Tojo, Y., Dairoku, H., Hasegawa, T., 2004. Reflexive orienting in response to eye gaze and an arrow in children with and without autism. J. Child Psychol. Psychiatry Allied Discip. 45 (3), 445-458. https://doi.org/10.1111/j.14697610.2004.00236.x

Sheridan, M.A., Sarsour, K., Jutte, D., D'Esposito, M., Boyce, W.T., 2012. The impact of social disparity on prefrontal function in childhood. PLoS One 7 (4), 35744. https:// doi.org/10.1371/journal.pone.0035744.

Shrout, P.E., Bolger, N., 2002. Mediation in experimental and nonexperimental studies: new procedures and recommendations. Psychol. Methods 7 (4), 422-445. https:// doi.org/10.1037/1082-989X.7.4.422.

Simons, D.J., Shoda, Y., Lindsay, D.S., 2017. Constraints on generality (COG): a proposed addition to all empirical papers. Perspect. Psychol. Sci. 12 (6), 1123-1128. https:// doi.org/10.1177/1745691617708630.

Summerfield, J.J., Lepsien, J., Gitelman, D.R., Mesulam, M.M., Nobre, A.C., 2006. Orienting attention based on long-term memory experience. Neuron 49 (6), 905-916. https://doi.org/10.1016/j.neuron.2006.01.021.

Tooley, U.A., Mackey, A.P., Ciric, R., Ruparel, K., Moore, T.M., Gur, R.C., Gur, R.E., Satterthwaite, T.D., Bassett, D.S., 2019. Associations between neighborhood SES and functional brain network development. Cereb. Cortex 30 (1), 1-19. https://doi.org/ 10.1093/cercor/bhz066.

Ungerleider, L.G., Haxby, J.V., 1994. "What" and "where" in the human brain. Curr. Opin. Neurobiol. 4 (2), 157-165. https://doi.org/10.1016/0959-4388(94)90066-3.

Ursache, A., Noble, K.G., 2016. Neurocognitive development in socioeconomic context: multiple mechanisms and implications for measuring socioeconomic status. Psychophysiology 53 (1), 71-82. https://doi.org/10.1111/psyp.12547.

Weiss, S.M., Meltzoff, A.N., Marshall, P.J., 2018. Neural measures of anticipatory bodily attention in children: relations with executive function. Dev. Cogn. Neurosci. 34 , 148-158. https://doi.org/10.1016/j.dcn.2018.08.002.

Wendelken, C., Baym, C.L., Gazzaley, A., Bunge, S.A., 2011. Neural indices of improved attentional modulation over middle childhood. Dev. Cogn. Neurosci. 1 (2), 175-186. https://doi.org/10.1016/j.dcn.2010.11.001.

Wu, T., Wang, X., Wu, Q., Spagna, A., Yang, J., Yuan, C., Wu, Y., Gao, Z., Hof, P.R., Fan, J., 2019. Anterior insular cortex is a bottleneck of cognitive control. NeuroImage 195, 490-504. https://doi.org/10.1016/j.neuroimage.2019.02.042.

Zelazo, P.D. (2006). The Dimensional Change Card Sort (DCCS): A method of assessing executive function in children. Nature Protocols. 1(1), 297-301. https://doi.org/10 .1038/nprot.2006.46.

Zhao, X., Lynch, J.G., Chen, Q., 2010. Reconsidering Baron and Kenny: myths and truths about mediation analysis. J. Consum. Res. 37 (2), 197-206. https://doi.org/ $10.1086 / 651257$. 